\title{
Disposable microfluidic micromixers for effective capture of Cryptosporidium parvum oocysts from water samples
}

\author{
L. Diéguez ${ }^{1,2^{*}}$ D, M. Winter ${ }^{1}$, S. Molan ${ }^{1}$, P. Monis ${ }^{1,3}$, B. King ${ }^{3}$ and B. Thierry ${ }^{1 *}$
}

\begin{abstract}
Background: Protecting drinking water supplies from pathogens such as Cryptosporidium parvum is a major concern for water utilities worldwide. The sensitivity and specificity of current detection methods are largely determined by the effectiveness of the concentration and separation methods used. The purpose of this study is to develop micromixers able to specifically isolate and concentrate Cryptosporidium, while allowing in situ analysis.

Results: In this study, disposable microfluidic micromixers were fabricated to effectively isolate Cryptosporidium parvum oocysts from water samples, while allowing direct observation and enabling quantification of oocysts captured in the device using high quality immunofluorescence microscopy. In parallel, quantitative analysis of the capture yield was carried out by analyzing the waste from the microfluidics outlet with an Imaging Flow Cytometer. At the optimal flow rate, capture efficiencies up to $96 \%$ were achieved in spiked samples.
\end{abstract}

Conclusions: Scaled microfluidic isolation and detection of Cryptosporidium parvum will provide a faster and more efficient detection method for Cryptosporidium compared to other available laboratory-scale technologies.

Keywords: Cryptosporidium parvum oocysts, Disposable microfluidic micromixers, Imaging flow cytometry, Water quality, Immunocytochemistry, Fluorescence microscopy

\section{Background}

Cryptosporidium is a highly resistant protozoan commonly encountered in surface waters. Although Cryptosporidium infections are self-limiting in healthy individuals, the consequences can be far more serious in infants and young children causing moderate to severe diarrhea [1]. Cryptosporidium outbreaks are often linked to treatment failures or treatment deficiencies at water treatment plants, allowing contamination of drinking water. Risk management of source waters requires cost effective, rapid and efficient monitoring of Cryptosporidium [2]. Procedures for Cryptosporidium detection typically includes collection of a large volume of water sample (10 - $1000 \mathrm{~L})$, followed by

\footnotetext{
* Correspondence: Iorena.dieguez@inl.int; benjamin.thierry@unisa.edu.au ${ }^{1}$ Future Industries Institute and ARC Centre of Excellence in Convergent Bio and Nano Science and Technology, University of South Australia, Mawson Lakes Campus, Mawson Lakes, South Australia, Australia

Full list of author information is available at the end of the article
}

concentration using various techniques including filtration and centrifugation to obtain a concentrated sample. The standardized procedure EPA 1623 describes a complete isolation/detection protocol based on filtration, elution from the filter and centrifugation to obtain a concentrated sample. The recovered sample then undergoes immunomagnetic separation to isolate oocysts from debris. Isolated oocysts are stained with specific fluorescent antibodies and nuclear stain for detection by immunofluorescence and microscopy. This method has demonstrated a recovery yield of $61 \%$ in spiked pure water, $48.8 \%$ in spiked filter tap samples and $19.5 \%$ in raw sources, with a limit of detection of approximately 10 oocysts/L [3]. The final steps in the EPA 1623 method require skilled technicians and are time- and resource-consuming. While immunofluorescence microscopy is the gold standard for the detection of the oocysts, other antigen based 
detection methods including ELISA (enzyme-linked immunosorbent assay) and immunochromatographic assays are also commercially available [4].

A number of alternative approaches have been reported to detect waterborne pathogens [5], including those based on Surface Plasmon Resonance [6], nucleic acid detection [7-11], immunocantilevers [12], Surface Enhanced Raman Scattering [13], dielectrophoresis [14] and impedance spectroscopy [15]. But all detection methods rely on efficient concentration of large volume of water sample and isolation of the pathogen. A number of microfabricated filters [1618] have been described and yielded oocysts capture efficiencies as high as $97 \%$ in spiked pure water samples. Microfluidic methods have also been actively investigated in recent years for oocysts detection due to their excellent reliability and efficiency. Owing to the inability of microfluidic systems to deal with very large volumes, these approaches have typically focused on treating samples that have already been subjected to the initial concentration step. Microfluidic filters achieved an efficiency of $86 \%$ when capturing Cryptosporidium oocysts from spiked concentrated water samples [19]. Inertial microfluidics has also been applied with some success to enrich water and food pathogens, yielding $100 \%$ efficiency sorting Cryptosporidium from concentrated water [20] and 68.4\% when recovering Giardia from food samples [21]. The Nano-DEP enrichment system provided a 10 times concentration of the raw sample [13]. These microfluidic systems rely on physical filtration or separation of oocysts from a pre-concentrated sample to deliver the sample for the final detection step. The pre-concentrated samples still need to be further processed to be analyzed using for example fluorescence microscopy. In addition, approaches based on physical features lack specificity in comparison to standard immunomagnetic separation. With this in mind, the integration of immunomagnetic separation with microfluidics has been advanced [22]. In addition, good recovery yield using microfabricated microwells functionalized with antibodies has also been reported, although the static nature of this approach limits its application to only a small volume of sample [23]. Recently, McGrath et al. reported on the interesting concept of high throughput Microfluidic Impedance Cytometry (MIC) for rapid enumeration and identification of different types of Cryptosporidium spiked in saline buffer and achieved over 92\% accuracy in discriminating Cryptosporidium parvum, Cryptosporidium muris and Giardia lamblia [24].

Microfluidic devices bioconjugated with specific molecular probes have been used with remarkable success in the isolation of circulating tumor cells from the blood of cancer patients [25]. To perform efficient immunocapture of cells in functionalized microfluidic devices, it is necessary to maximize the surface to volume ratio and to create appropriate mixing to optimize the chances of the target cells coming into contact with the immunoconjugated surface. To our knowledge, the application of a biofunctionalised micromixer for the immunospecific capture of Cryptosporidium has not been investigated thus far. Towards simplifying the workflow of Cryptosporidium oocysts detection from a pre-concentrated water sample with increasing accuracy, the aim of this work is to evaluate a streamlined microfluidic technology for its ability to specifically isolate Cryptosporidium oocysts from concentrated water samples while simultaneously allowing for high quality immunofluorescence observation. In this way, oocysts present in pre-concentrated samples (using any of the above mentioned approaches) can be specifically isolated and quantified in a single system. A positive enrichment strategy has been used due to sample/technical specificities including multiple contamination source in water samples and the existence of an antibody that binds the target oocysts with high specificity and affinity. For this purpose, disposable microfluidic devices were fabricated and functionalized with an antibody (Cry 104) specific against antigens expressed on the oocyst surface. The microfluidic device consists of an array of $25 \mu \mathrm{m}$ thin micromixers designed to enhance the surface interactions between the Cryptosporidium and the channel walls. Once isolated within the micromixer, the oocysts can be readily stained using standard fluorescent tags and observed in situ under fluorescence microscopy, providing a simpler alternative to the current method based on immunomagnetic separation. Under optimal conditions, a recovery yield of up to $96 \%$ could be obtained for Cryptosporidium oocysts spiked in water.

The design of this microfluidic device enhances mixing and hence binding of the Cryptosporodium with its many parallel channels which not only increase the area for binding but also provides some redundancy for blockage as would be expected with environmental water.

\section{Methods}

The micromixers were fabricated in SU8 on a silicon wafer. The design consists of an array of $25 \mu \mathrm{m}$ high microchannels favoring chaotic mixing. Standard soft lithography was used to produce disposable microfluidic devices, sealed with oxygen plasma. Using a silane-based functionalization strategy, antibodies were immobilized on the channel surface. Known numbers of Cryptosporidium were spiked in saline buffer and introduced through the microfluidic devices at different flow rates. The oocysts captured in the device were washed, fixed, permeabilized and stained prior to microscope examination. The waste solution recovered from the devices was also analyzed with an Imaging Flow Cytometer Image Stream X (ISX, AMNIS, Seattle, WA, USA). 


\section{Materials}

Phosphate buffered saline (PBS), (3-aminopropyl) trimethoxysilane (APTMS), trichloro 1,1,2,2-perfluorooctyl-silane, bovine serum albumin (BSA), fetal bovine serum (FBS), glutaraldehyde, formaldehyde, $\operatorname{Triton}^{\mathrm{Tm}} \mathrm{X}-100$, and 4',6-Diamidino-2-phenylindole dihydrochloride (DAPI) were purchased from Sigma Aldrich (USA). $\gamma$-irradiated Cryptosporidium parvum were kindly donated from SA Water (Australia). Specific monoclonal antibody Cry104 was obtained from BTF Biomerieux (Australia). A FITC goat anti mouse IgG secondary antibody was purchased from Sigma Aldrich (USA). Polydimethylsiloxane (PDMS) elastomer SYLGARD 184 was obtained from Dow Corning (USA) and SU-8 10 photoresist was purchased from MicroChem (USA). All other chemicals were analytical grade. Silicon wafers with a 3" diameter were obtained from Micro Materials \& Research Cons. Pty Ltd (Australia), and the syringe pumps KDS-212-CE and KDS-210 used in this study were purchased from KD Scientific.

\section{Fabrication of microfluidic devices}

Standard photolithography was used to fabricate chaotic mixing silicon masters for PDMS molding, as previously described [26]. Briefly, silicon substrates were cleaned with acetone in a sonic bath for $5 \mathrm{~min}$ and then with isopropanol for $5 \mathrm{~min}$. After rinsing with isopropanol, substrates were dried with a nitrogen gun and activated with oxygen plasma for $10 \mathrm{~min}$. The negative photoresist, SU-8 10, was then spin-coated onto the wafer. The SU-8 was then patterned using a film photomask (JD tools) with a UV dose of $225 \mathrm{~mJ} / \mathrm{cm}^{2}$ and postbaked at $65{ }^{\circ} \mathrm{C}$ for $1 \mathrm{~min}$ and at $95{ }^{\circ} \mathrm{C}$ for $2 \mathrm{~min}$. SU- 8 was then developed during $2 \mathrm{~min}$ to form a template and hard-baked with a ramping temperature from $65{ }^{\circ} \mathrm{C}$ to $200{ }^{\circ} \mathrm{C}$.

The template was hydrophobized submitting it to a trichloro 1,1,2,2-perfluorooctyl-silane vapor in a desiccator for $1 \mathrm{~h}$ at $80{ }^{\circ} \mathrm{C}$, and covered with liquid PDMS (the PDMS prepolymer was mixed with the crosslinker at a 10:1 ratio and degassed). PDMS was then degassed, cured at $80{ }^{\circ} \mathrm{C}$ for $1 \mathrm{~h}$ and unmolded from the silicon master. Inlet and outlet were punched in the PDMS replica that was then irreversibly sealed against a clean glass slide upon treatment in oxygen plasma at low power for $15 \mathrm{~s}$.

\section{Functionalization of the microfluidic devices}

After attaching tubing to the inlet and outlet ports, the micromixers were connected to a syringe pump and filled with ethanol at a flow rate of $100 \mu \mathrm{l} / \mathrm{min}$. Once the devices were stabilized, 2\% APTMS in ethanol was withdrawn into the device for $30 \mathrm{~min}$ and rinsed with ethanol for $10 \mathrm{~min}$. The buffer solution was then changed with MilliQ water and stabilized for $10 \mathrm{~min}$ prior to withdrawal of $1 \%$ glutaraldehyde in water for another $30 \mathrm{~min}$ and rinsing in ultrapure water for $10 \mathrm{~min}$. PBS was then withdrawn into the device and equilibrated for $10 \mathrm{~min}$ just before introducing $200 \mu \mathrm{l}$ of $50 \mu \mathrm{g} / \mathrm{ml} \mathrm{Cry104}$ in PBS that was left to react overnight at $4{ }^{\circ} \mathrm{C}$. Unreacted antibodies were rinsed with $\mathrm{PBS}$ and the surface blocked with $2 \%$ BSA in PBS. All the functionalization steps were done at the same flow rate. Control devices were functionalized following the same protocol, but without the antibody conjugation.

\section{Capture of Cryptosporidium parvum oocysts}

To optimize the parameters for the isolation of Cryptosporidium, small volumes $(50 \mu \mathrm{l})$ of high concentrated spiked samples in PBS $\left(1.5 \times 10^{6}\right.$ and $1.5 \times 10^{4}$ oocysts $/ \mathrm{ml}$ ) were injected into the functionalized micromixer at flow rates of $0.5,2$ or $5 \mu \mathrm{l} / \mathrm{min}$ to allow binding of the oocysts to the specific antibodies and explore the effect of flow rate. Washing of unbound oocysts was conducted at the same flow as for binding of oocysts, i. e. $0.5,2$ or $5 \mu \mathrm{l} / \mathrm{min}$.

\section{Fluorescence microscopy studies}

The microfluidic devices were examined under a Nikon $\mathrm{Ti}$ Eclipse inverted fluorescence microscope. The oocysts isolated in the device were fixed with $4 \%$ formaldehyde, permeabilised with $0.05 \%$ Triton X-100 and stained with 1:10,000 DAPI at $0.5,2$ or $5 \mu \mathrm{l} / \mathrm{min}$ (same as flow rate used for oocyte binding) to allow identification by fluorescence microscopy as a proof of concept. The presence of DAPI fluorescent bodies identified as oocysts captured inside the device was quantified in situ by imaging 50 randomly chosen low magnification fields of view in different sections of the device, using a $10 \times$ objective.

Towards demonstrating the relevance of this approach for environmental samples, the presence of Cryptosporidium oocysts was also confirmed using the Cry104 antibody. Briefly, after capture, the device was incubated with the Cry104 antibody (1/50) for $60 \mathrm{~min}$. After washing with PBS, permeabilisation and blocking was performed with $0.05 \%$ Triton X-100 and $2 \%$ BSA for $10 \mathrm{~min}$. The devices were then incubated with the FITC goat anti mouse IgG secondary antibody for $30 \mathrm{~min}$ as per the manufacturer's instructions, before washing and imaging.

\section{Imaging flow cytometry studies}

To quantitatively characterise the performance of the device and calculate the isolation yield of the micromixer, the input solution and the solution eluted from the device were recovered and analyzed with an Imaging Flow Cytometer (IFC) Image Stream X (AMNIS, Seattle, 
WA, USA). Solutions were centrifuged at 10,000 RCF for $15 \mathrm{~min}$ at $4{ }^{\circ} \mathrm{C}$. The supernatants were removed and the pellets resuspended to $100 \mu \mathrm{l}$ for Imaging Flow analysis. Since Cryptosporidium particles were already stained with DAPI during the microfluidic procedure, channel 1 of the flow cytometer was set to record all the DAPI stained events, with the 405 laser. Channel 4 and 6 were set for brightfield and darkfield images, respectively. In order to discriminate the oocysts from autofluorescent debris, a size classification was applied.

To confirm that the DAPI-stained bodies identified as oocysts were indeed Cryptosporidium parvum, the recovered sample was labelled with the specific Cry104 antibody and a goat anti mouse FITC IgG secondary antibody was used. Briefly, after blocking with $10 \%$ fetal bovine serum in PBS for $10 \mathrm{~min}$ on ice, the sample was incubated with the Cry104 antibody (1/50) for $60 \mathrm{~min}$. After washing with PBS, $0.05 \%$ Triton X-100 and 1\% FBS, the sample was incubated with the FITC secondary antibody (1/5000) for $30 \mathrm{~min}$. After washing with PBS samples were run through the IFC. The FITC events were recorded in Channel 2 of the IFC with the 488 laser. Controls were performed to discriminate nonspecific staining.

The capture efficiency was calculated based on eq. 1.

\section{Capture Efficiency (\%)$$
=\frac{(\# \text { oocytes introduced })-(\# \text { oocytes } \text { in waste })}{(\# \text { oocytes introduced })} \times 100
$$

\section{Statistical analysis}

Statistical analysis was performed in Matlab (2017b, Mathworks) and Excel. Student's t-tests were used for directly comparing two variables. A 1-way ANOVA (confidence interval 0.05) adjusted using Bonferroni correlation was used for multi-variable analysis.

\section{Results and discussion}

Capture of Cryptosporidium in the micromixer microfluidic devices

To investigate the effect of the velocity on the capture efficiency, $50 \mu \mathrm{l}$ of Cryptosporidium oocyst solutions were initially flowed at a high concentration (1.5 $\times$ $10^{6}$ oocysts / mL, $\sim 75,000$ oocysts) into the micromixers under 3 different flow rates. Microfluidic micromixers conjugated with BSA instead of the anti- Cryptosporidium antibody were used as controls. All experiments were performed in duplicate. IFC was used as a tool to quantitatively determine the capture efficiency of Cryptosporidium in the microfluidic micromixers. To this end, the unbound oocysts were recovered from the outlet of the microfluidic devices and prepared for

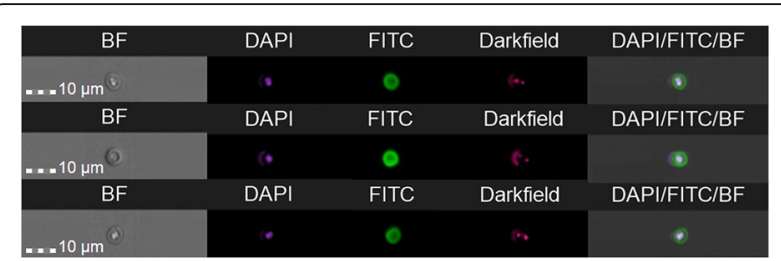

Fig. 1 Cryptosporidium images from the different channels in the Imaging Flow Cytometer: Brightfield (BF), Darkfield, nuclear staining in DAPI and the Cryptosporidium specific Cry104 antibody (goat anti mouse FITC IgG secondary antibody), and the combined image. Images taken at 400x magnification and have been adjusted to enhance visual appearance

analysis with IFC. Figure 1 shows Cryptosporidium oocysts recorded from different channels in the IFC: brightfield, DAPI, and Cry104 antibody labelled with a goat anti mouse FITC IgG secondary antibody, brightfield and darkfield.

Once the protocol to identify and count the oocysts with IFC was optimized, the capture efficiency for each flow rate was calculated. At $0.5 \mu \mathrm{L} / \mathrm{min}$, the average capture efficiency was $92 \%$, a maximum average capture efficiency of $96 \%$ was determined at $2 \mu \mathrm{L} / \mathrm{min}$ - only 3375 of the initial 75,000 oocysts were counted in the device's waste (Fig. 2). There was statistical differences between bioconjugated and unconjugated control chip capture efficiencies for both 0.5 and $2 \mu \mathrm{l} / \mathrm{min}$ (students $t$-test, $P<0.05)$. There was, however, no difference in the capture efficiency between the 0.5 and $2 \mu \mathrm{l} / \mathrm{min}$ flow rates for the functionalised device. On the other hand, increased flow rates led to drastic decreases in the recovery yield with only $40 \%$ of the oocysts being recovered at $5 \mu \mathrm{l} / \mathrm{min}$ (45,225 in the waste). There was no statistical difference in capture between the $5 \mu \mathrm{l} / \mathrm{min}$ experiment and its respective control. This is due to the specific design of the F-shape micromixer, which maximizes mixing at lower flow rates. All control experiments resulted in a low non-specific capture of the oocysts within the non biofunctionalized micromixers $(18 \%, 10 \%$ and $9 \%$ for $0.5,2$ and $5 \mu \mathrm{L} / \mathrm{min}$, respectively).

The use of IFC for characterization of the device performance is highly relevant, since it provides excellent quality of images for the micron-scale Cryptosporidium as well as the required high throughput quantification of the eluted samples. Standard flow cytometry, in contrast with IFC, encounters problems with the presence of auto fluorescent debris and clumps of Cryptosporidium, inhibiting proper identification and therefore, quantification [27]. The quantitative data obtained using IFC was confirmed using fluorescence microscopy (Fig. 3) of the micromixers. In good agreement with IFC, the highest number of Cryptosporidium oocysts retained in the 


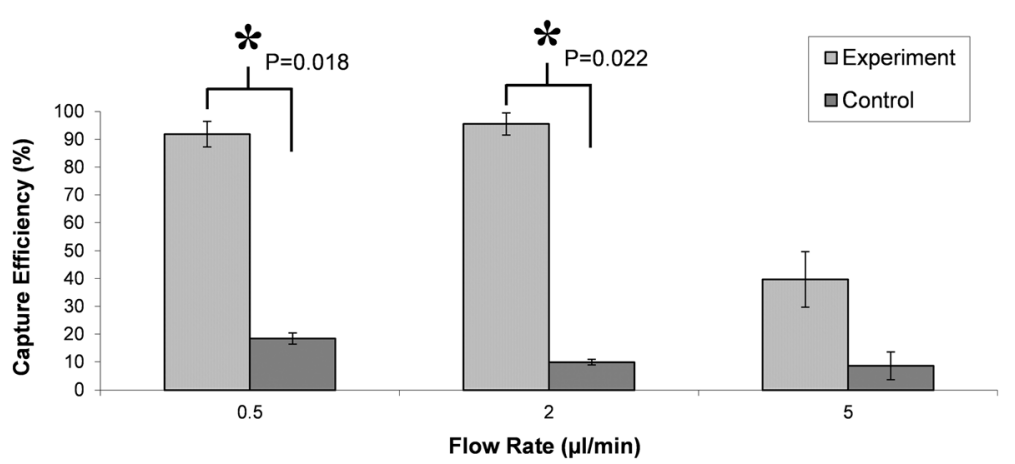

Fig. 2 Capture efficiency of Cryptosporidium oocysts in the microfluidic devices at different flow rates calculated with Imaging Flow Cytometry. Light grey bars are the functionalized experimental results and the dark grey bars are the non-functionalized control devices. The average capture efficiency was 92\%, 96\% and 40\% at 0.5, 2 and $5 \mu \mathrm{l} / \mathrm{min}$ respectively. Capture efficiency in the control devices was 18\%, 10\% and 9\% for 0.5, 2 and $5 \mu \mathrm{L} / \mathrm{min}$, respectively

micromixer was at the operating flow-rate of $2 \mu \mathrm{l} / \mathrm{min}$. Minimal non-specific binding in control (non-functionalized) devices was also observed, confirming the specific nature of the binding. A flow rate of $2 \mu \mathrm{l} / \mathrm{min}$ was thus chosen to enhance throughput of the system without compromising capture efficiency.

An important feature of the proposed microfluidic micromixer approach is the possibility to carry out high quality imaging directly within the devices themselves. Standard staining of the captured Cryptosporidium oocysts with the specific antibody Cry104 and DAPI, enabled straightforward visualization of the oocysts inside the micromixer as shown in Fig. 4.

The oocysts had a strong binding affinity to the antibody-functionalized micromixers as shown by preferential binding towards the inlet side (Fig. 5a). To confirm this observation, the number of oocysts captured at different lengths in the device was systematically counted using the following protocol: the number of oocysts captured was counted at 10 randomly chosen areas at different lengths into the device $(0,4,8,12$ and $16 \mathrm{~mm}), 0$ and 16 being the inlet and the outlet of the micromixer, respectively. Then the number of oocysts per area was averaged at each length and plotted in Fig. 5b. For each position there was statistically more oocysts captured in the Cry104 functionalised device than in the control $(p<0.05$, students $t$-test $)$. It was observed that the number of oocysts found in the microfluidic device is statistically different across the device decreasing with the penetration length with most binding occurring in the first part of the device (F statistic 0.0012, 1-way ANOVA). Specifically, at $0 \mathrm{~mm}$ penetration into the device there are statistically more Cryptosporidium than at 12 and $16 \mathrm{~mm}$. For the control there was no difference in binding between any of the positions ( $\mathrm{F}$ statistic 0.1039, 1-way ANOVA). This clearly demonstrates that Cryptosporidium oocysts have specific and strong binding affinity to the antibody bioconjugated device.

To further confirm the validity of our system to isolate and quantify Cryptosporidium presence in water samples,

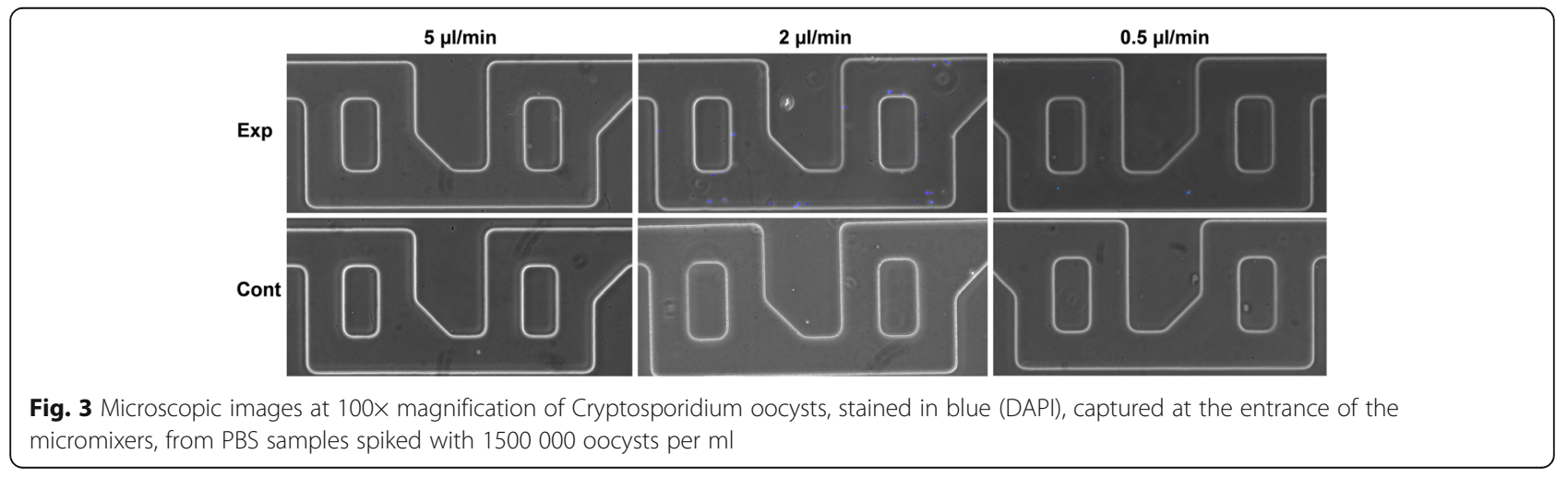



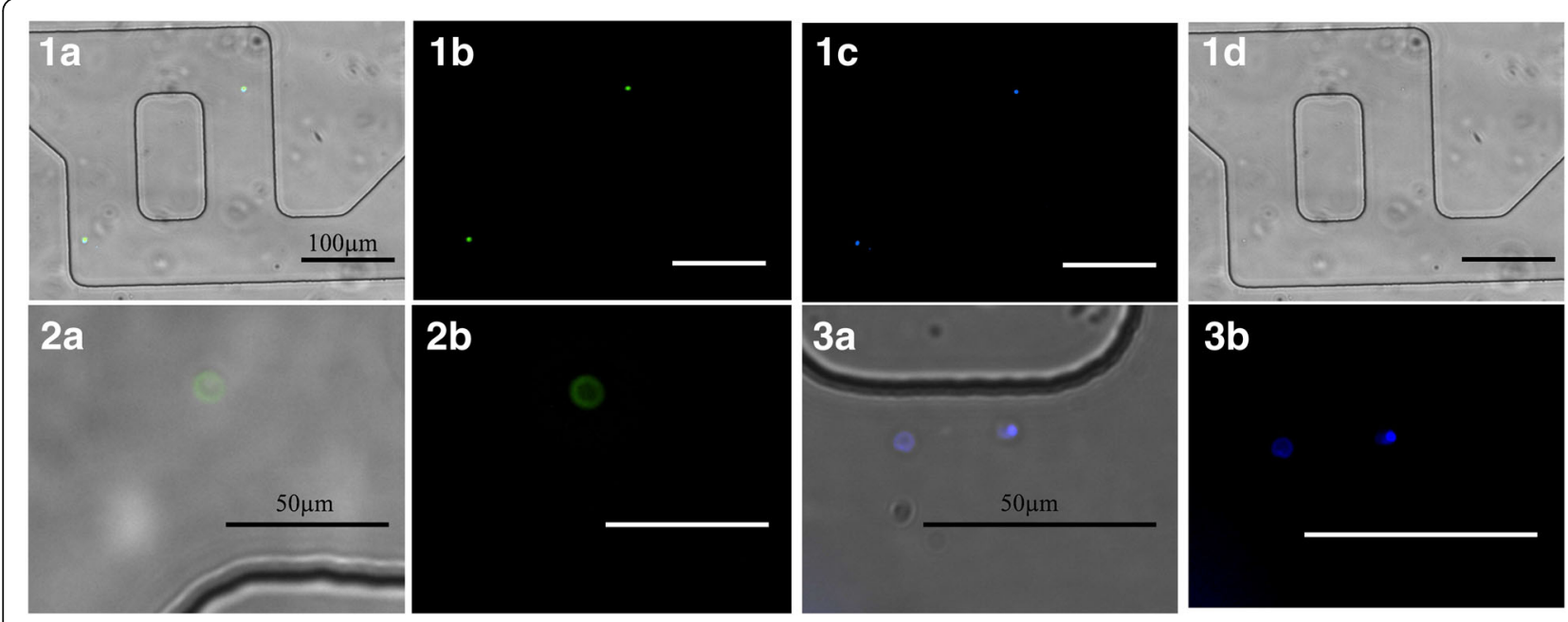

Fig. 4 Microscopic images of the Cryptosporidium oocysts captured inside the functionalized microfluidic devices at the flow rate of $2 \mu \mathrm{l} / \mathrm{min}$. Cryptosporidium oocysts are specifically recognized by Cry 104 antibody, stained with a secondary FITC antibody and imaged at 100x, scale bar 100 um (1). Composite image with brightfield (BF), FITC and DAPI channels (1a), DAPI channel (1b), FITC channel (1c) and BF image (1d). Images were also taken at higher magnification, 400x, scale bar $50 \mu \mathrm{m}(\mathbf{2}, \mathbf{3})$. Composite images with BF and FITC (2a) or BF and DAPI (3a), and individual FITC channel (2b), and DAPI channel (3b)

the capture efficiency of Cryptosporidium oocysts was also studied at a lower concentration. For this purpose, $50 \mu \mathrm{l}$ of two different concentrations of Cryptosporidium, $1.5 \times$ $10^{6}$ and $1.5 \times 10^{4}$ oocysts $/ \mathrm{ml}(75,000$ and 750 oocysts, respectively), were introduced at $2 \mu \mathrm{l} / \mathrm{min}$, in the micromixers and the mean capture efficiencies were determined with IFC. As shown in Fig. 6, the capture efficiency remains constant independently of the Cryptosporidium concentration. For both, 75,000 and 750, there was a statistical difference between the functionalised device and the control $(p<0.05$, students, $t$-test $)$, with significally more binding in the functionalised device.

Once the ability of the system to capture and detect Cryptosporidium oocytes has been confirmed, for the system to be an analytical tool for quantification, a counting protocol needs to be defined. For this purpose, a calibration curve needs to be established to relate the number of oocytes counted by fluorescence microscopy to the real

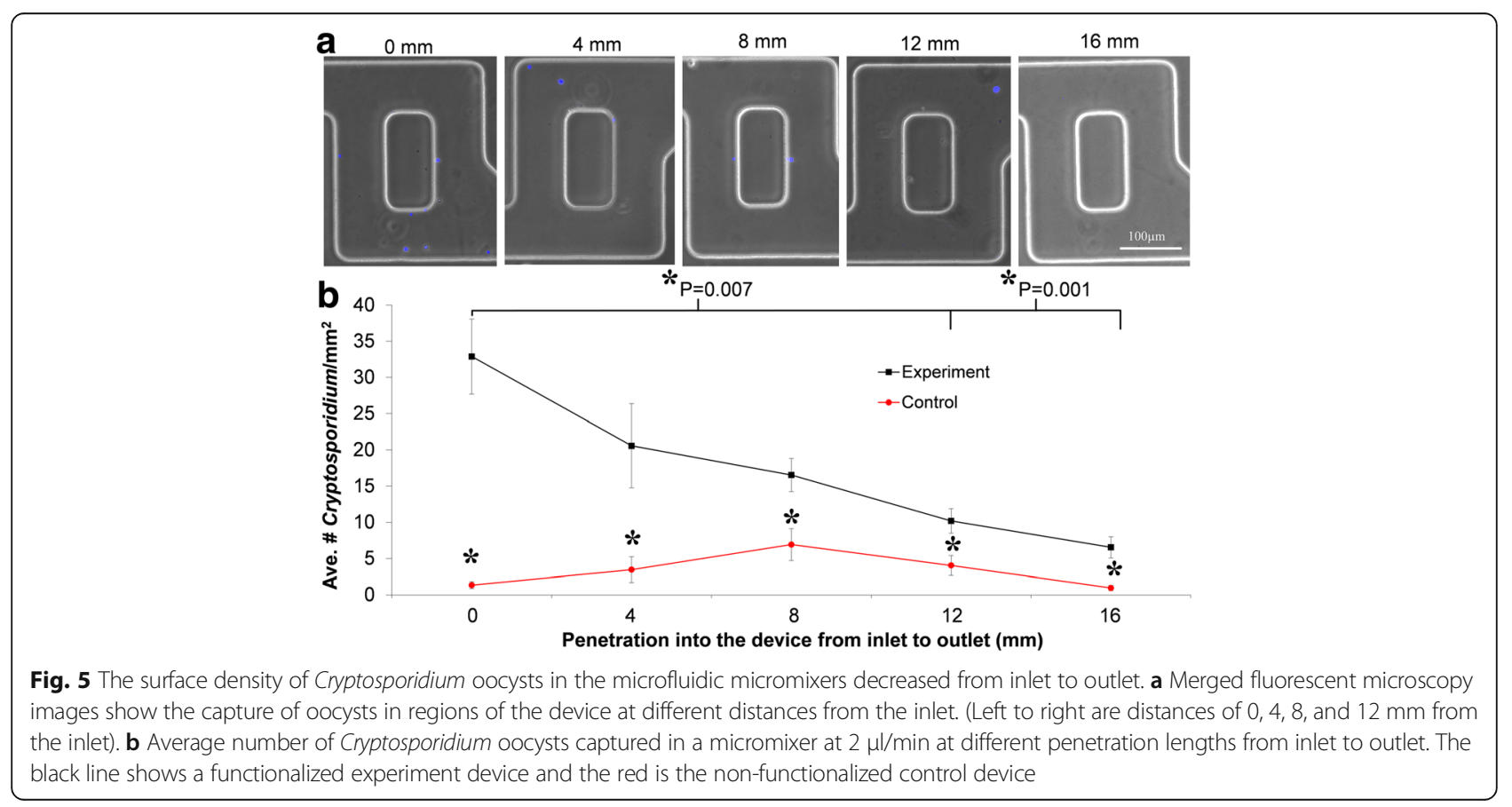




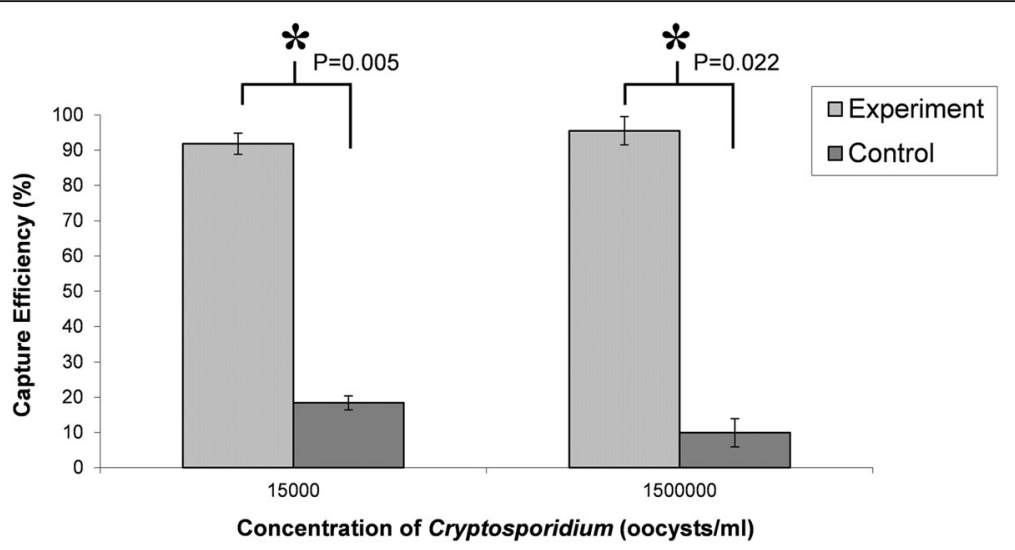

Fig. 6 Dependence of the capture efficiency of Cryptosporidium oocysts in microfluidic micromixers at $2 \mu \mathrm{l} / \mathrm{min}$ at concentrations 15,000 and 1,500,000 crypto/ml, using imaging flow cytometry. Light grey bars are the functionalized experimental results and the dark grey bars are the non-functionalized control devices

concentration of a given tested sample. Similarly to Fig. 5, we propose that the number of oocysts captured at different lengths in the device should be systematically counted at several randomly chosen areas. Then the average number of oocysts per area at different lengths could be plotted and fitted to a classical kinetics exponential decay, as in eq. 2 :

$$
y(x)=A e^{-k x}
$$

where $y(x)$ is the average number of oocytes in the device at a given length $x$. Following this protocol at different initial concentrations of Cryptosporidium and normalizing $y(x)$, a calibration curve could be theoretically established for the dynamic range between the constant of decay $\mathrm{k}$ and the concentration of the solution. Alternatively, if the number of oocysts is very low, the total area of the microfluidic device can be directly scanned to find the concentration.

\section{Conclusion}

The detection of pathogenic oocysts in water is an important health and environmental issue. A microfluidic micromixer device was developed to capture Cryptosporidium and enable direct in situ high quality microscopic observation. Imaging flow cytometry and fluorescence microscopy demonstrated that the oocysts had a strong binding affinity to the antibody-functionalized micromixers. Only minimal non-specific binding was observed in control devices, confirming the specific nature of the binding. A capture efficiency of $96 \%$ was determined under optimal conditions. Further studies are warranted beyond this proof of principle work to validate the proposed approach for its analytical potential for quantification in real samples. The main limitation of this microfluidic technology is its limited throughput, which restricts its application to water concentrates. However, it is anticipated that a minor redesign and/or multiplexing of the microfluidic mixers could lead to higher throughputs that would be readily compatible with volumes of water typically obtained from standard concentration methods as described in the EPA guideline. Besides its excellent efficiency, the main advantage of this technology is that its application would have strong potential to simplify the overall workflow, leading to notable time and resource savings. In summary, the microfluidic micromixer approach can capture Cryptosporidium in water concentrates and has the potential to accelerate and simplify the detection of Cryptosporidium and other microorganisms of concern in surface or drinking water.

\section{Abbreviations}

APTMS: (3-aminopropyl) trimethoxysilane; BSA: Bovine Serum Albumin; DAPI: 4',6-Diamidino-2-phenylindole dihydrochloride; ELISA: Enzyme-Linked Immunosorbent Assay; FBS: Fetal Bovine Serum; IFC: Imaging Flow

Cytometer; MIC: Microfluidic Impedance Cytometry; PBS: Phosphate Buffered Saline; PDMS: Polydimethylsiloxane

\section{Acknowledgements}

This work was performed in part at the South Australian node of the Australian National Fabrication Facility, a company established under the National Collaborative Research Infrastructure Strategy to provide nano and micro-fabrication facilities for Australia's researchers.

\section{Funding}

This work has been supported by the NHMRC Project grant APP1045841 and by the NORTE-45-2015-02 program under grant NORTE-01-0145-FEDER-000029. Thierry is supported by a NHMRC CDA Fellowship.

\section{Availability of data and materials}

The datasets used and/or analysed during the current study are available from the corresponding author on reasonable request.

\section{Authors' contributions}

LD designed and fabricated the microfluidic masters and devices, designed the surface functionalization strategy and the experimental protocol for cryptosporidium isolation. She was involved in the planning of all experiments and was the primary responsible of manuscript writing. MW participated in the fluorescent labelling of the cryptospodirium, was in charge of the imaging flow cytometry and participated extensively in 
manuscript writing. SM fabricated the disposable PDMS microfluidic devices, and carried out all the experiments for the optimisation of oocytes isolation, labelling and quantification. PM and BK contributed to the design of the isolation strategy, provided the irradiated oocytes and developed the antibody used for the isolation. They also participated in the manuscript writing. BT directed the work, had the initial idea of the project and followed closely the experimental procedures. He also supported the work financially and was involved in the manuscript writing. All authors read and approved the final manuscript.

\section{Ethics approval and consent to participate} Not applicable.

\section{Consent for publication}

Not applicable.

\section{Competing interests}

The authors declare that they have no competing interests.

\section{Publisher's Note}

Springer Nature remains neutral with regard to jurisdictional claims in published maps and institutional affiliations.

\section{Author details}

${ }^{1}$ Future Industries Institute and ARC Centre of Excellence in Convergent Bio and Nano Science and Technology, University of South Australia, Mawson Lakes Campus, Mawson Lakes, South Australia, Australia. ${ }^{2}$ International Iberian Nanotechnology Laboratory, Braga, Portugal. ${ }^{3}$ South Australian Water Corporation, Adelaide, SA, Australia.

\section{Received: 7 November 2017 Accepted: 14 March 2018}

Published online: 27 March 2018

\section{References}

1. Kotloff KL, Nataro JP, Blackwelder WC, Nasrin D, Farag TH, Panchalingam S, et al. Burden and aetiology of diarrhoeal disease in infants and young children in developing countries (the global enteric multicenter study, GEMS): a prospective, case-control study. Lancet. 2013;382:209-22. https://doi.org/10.1016/S0140-6736(13)60844-2.

2. Betancourt WQ, Rose JB. Drinking water treatment processes for removal of Cryptosporidium and Giardia. Vet Parasitol. 2004;126:219-34. https://doi.org/10.1016/j.vetpar.2004.09.002.

3. Carey CM, Lee $\mathrm{H}$, Trevors JT. Biology, persistence and detection of Cryptosporidium parvum and Cryptosporidium hominis oocyst. Water Res. 2004;38:818-62. https://doi.org/10.1016/j.watres.2003.10.012.

4. Checkley W, White AC, Jaganath D, Arrowood MJ, Chalmers RM, Chen XM, et al. A review of the global burden, novel diagnostics, therapeutics, and vaccine targets for cryptosporidium. Lancet Infect Dis. 2015;15:85-94. https://doi.org/10.1016/S1473-3099(14)70772-8.

5. Bridle H, Kersaudy-Kerhoas M, Miller B, Gavriilidou D, Katzer F, Innes EA, et al. Detection of Cryptosporidium in miniaturised fluidic devices. Water Res. 2012;46:1641-61. https://doi.org/10.1016/j.watres.2012.01.010.

6. Kang CD, Lee SW, Park TH, Sim SJ. Performance enhancement of real-time detection of protozoan parasite, Cryptosporidium oocyst by a modified surface plasmon resonance (SPR) biosensor. Enzym Microb Technol. 2006;39: 387-90. https://doi.org/10.1016/j.enzmictec.2005.11.039.

7. Crannell ZA, Castellanos-Gonzalez A, Irani A, Rohrman B, White AC, Richards-Kortum R. Nucleic acid test to diagnose cryptosporidiosis: lab assessment in animal and patient specimens. Anal Chem. 2014;86:2565-71. https://doi.org/10.1021/ac403750z.

8. Srinivasan V, Stedtfeld RD, Tourlousse DM, Baushke SW, Xin Y, Miller SM, et al. Diagnostic microarray for 14 water and foodborne pathogens using a flatbed scanner. J Microbiol Methods. 2017;139:15-21. https://doi.org/10.1016/j.mimet.2017.04.009

9. Gómez-de Pedro S, Berenguel-Alonso M, Couceiro P, Alonso-Chamarro J Puyol M. Automatic microfluidic system to perform multi-step magneto-biochemical assays. Sensors Actuators B Chem. 2017;245:477-83. https://doi.org/10.1016/j.snb.2017.01.158.

10. Deshmukh RA, Joshi K, Bhand S, Roy U. Recent developments in detection and enumeration of waterborne bacteria: a retrospective minireview. Microbiology. 2016;5:901-22. https://doi.org/10.1002/mbo3.383.
11. Stokdyk JP, Firnstahl AD, Spencer SK, Burch TR, Borchardt MA. Determining the $95 \%$ limit of detection for waterborne pathogen analyses from primary concentration to qPCR. Water Res. 2016;96:105-13. https://doi.org/10.1016/j. watres.2016.03.026.

12. Bridle H, Wang W, Gavriilidou D, Amalou F, Hand DP, Shu W. Static mode microfluidic cantilevers for detection of waterborne pathogens. Sensors Actuators A Phys. 2016;247:144-9. https://doi.org/10.1016/j.sna.2016.05.011.

13. Wang C, Madiyar F, Yu C, Li J. Detection of extremely low concentration waterborne pathogen using a multiplexing self-referencing SERS microfluidic biosensor. J Biol Eng. 2017;11:9. https://doi.org/10.1186/ s13036-017-0051-x

14. Goater AD, Burt JPH, Pethig R. A combined travelling wave dielectrophoresis and electrorotation device: applied to the concentration and viability determination of Cryptosporidium. J Phys D Appl Phys. 1997;30:L65-9. https://doi.org/10.1088/0022-3727/30/18/001.

15. Houssin T, Follet J, Follet A, Dei-Cas E, Senez V. Label-free analysis of waterpolluting parasite by electrochemical impedance spectroscopy. Biosens Bioelectron. 2010;25:1122-9. https://doi.org/10.1016/J.BIOS.2009.09.039.

16. Taguchi T, Arakaki A, Takeyama H, Haraguchi S, Yoshino M, Kaneko M, et al. Detection ofCryptosporidium parvum oocysts using a microfluidic device equipped with the SUS micromesh and FITC-labeled antibody. Biotechnol Bioeng. 2007;96:272-80. https://doi.org/10.1002/bit.21104.

17. Warkiani M, Chen L, Lou C, Liu H, Zhang R, Gong H. Capturing and recovering of Cryptosporidium parvum oocysts with polymeric microfabricated filter. J Memb Sci. 2011;369:560-8. https://doi.org/10.1016/j. memsci.2010.12.038

18. Pires NMM, Dong T. A cascade-like silicon filter for improved recovery of oocysts from environmental waters. Environ Technol. 2014;35:781-90. https://doi.org/10.1080/09593330.2013.851280.

19. Kim J, Erath J, Rodriguez A, Yang C. A high-efficiency microfluidic device for size-selective trapping and sorting. Lab Chip. 2014;14:2480-90. https://doi. org/10.1039/C4LC00219A.

20. Jimenez M, Miller B, Bridle HL. Efficient separation of small microparticles at high flowrates using spiral channels: application to waterborne pathogens. Chem Eng Sci. 2017;157:247-54. https://doi.org/10.1016/j.ces.2015.08.042.

21. Ganz KR, Clime L, Farber JM, Corneau N, Veres T, Dixon BR. Enhancing the detection of Giardia duodenalis cysts in foods by inertial microfluidic separation. Appl Environ Microbiol. 2015;81:3925-33. https://doi.org/10. 1128/AEM.03868-14.

22. Ramadan Q, Gijs MAM. Microfluidic applications of functionalized magnetic particles for environmental analysis: focus on waterborne pathogen detection. Microfluid Nanofluidics. 2012;13:529-42. https://doi.org/10.1007/s10404-012-1041-4.

23. Taguchi T, Takeyama H, Matsunaga T. Immuno-capture of Cryptosporidium parvum using micro-well array. Biosens Bioelectron. 2005;20:2276-82. https://doi.org/10.1016/j.bios.2004.10.017.

24. McGrath JS, Honrado C, Spencer D, Horton B, Bridle HL, Morgan H. Analysis of parasitic Protozoa at the single-cell level using microfluidic impedance cytometry. Sci Rep. 2017;7:2601. https://doi.org/10.1038/s41598-017-02715-y.

25. Nagrath S, Sequist LV, Maheswaran S, Bell DW, Irimia D, Ulkus L, et al. Isolation of rare circulating tumour cells in cancer patients by microchip technology. Nature. 2007;450:1235-9. https://doi.org/10.1038/nature06385.

26. Diéguez L, Winter MA, Pocock KJ, Bremmell KE, Thierry B. Efficient microfluidic negative enrichment of circulating tumor cells in blood using roughened PDMS. Analyst 2015;140:3565-3572. https://doi.org/10.1039/ C4AN01768D

27. Hsu BM, Wu NM, Jang HD, Shih FC, Wan MT, Kung CM. Using the flow cytometry to quantify the giardia cysts and cryptosporidium oocysts in water samples. Environ Monit Assess. 2005;104:155-62. https://doi.org/10. 1007/s10661-005-1608-6. 